Open Access

\title{
Increased mortality in elderly patients with acute respiratory distress syndrome is not explained by host response
}

Laura R. A. Schouten ${ }^{1,2,3^{*}}$ (D), Lieuwe D. J. Bos ${ }^{2,3}$, A. Serpa Neto ${ }^{2,4}$, Lonneke A. van Vught ${ }^{5}$, Maryse A. Wiewel ${ }^{5}$, Arie J. Hoogendijk, Marc J. M. Bonten ${ }^{6}$, Olaf L. Cremer ${ }^{7}$, Janneke Horn²,3, Tom van der Poll ${ }^{5}$, Marcus J. Schultz ${ }^{2,3,8}$, Roelie M. Wösten-van Asperen ${ }^{9}$ and the MARS consortium

\footnotetext{
*Correspondence: I.r.schouten@ amsterdamumc.nl ${ }^{1}$ Department of Pediatric Intensive Care, Academic Medical Center, University of Amsterdam, Amsterdam, the Netherlands ${ }^{2}$ Department of Intensive Care, Academic Medical Center, University of Amsterdam, Amsterdam, The Netherlands Full list of author information is available at the end of the article
}

\begin{abstract}
Background: Advanced age is associated with increased mortality in acute respiratory distress syndrome (ARDS) patients. Preclinical studies suggest that the host response to an injurious challenge is age-dependent. In ARDS patients, we investigated whether the association between age and mortality is mediated through age-related differences in the host response.
\end{abstract}

Methods: This was a prospective longitudinal observational cohort study, performed in the ICUs of two university-affiliated hospitals. The systemic host response was characterized in three predefined age-groups, based on the age-tertiles of the studied population: young (18 to 54 years, $N=209$ ), middle-aged (55 to 67 years, $N=213$ ), and elderly (67 years and older, $N=196$ ). Biomarkers of inflammation, endothelial activation, and coagulation were determined in plasma obtained at the onset of ARDS. The primary outcome was 90-day mortality. A mediation analysis was performed to examine whether age-related differences in biomarker levels serve as potential causal pathways mediating the association between age and mortality.

Results: Ninety-day mortality rates were 30\% (63/209) in young, 37\% (78/213) in middle-aged, and 43\% (84/196) in elderly patients. Middle-aged and elderly patients had a higher risk of death compared to young patients (adjusted odds ratio, 1.5 [95\% confidence interval 1.0 to 2.3] and 2.1 [1.4 to 3.4], respectively). Relative to young patients, the elderly had significantly lower systemic levels of biomarkers of inflammation and endothelial activation. Tissue plasminogen activator, a marker of coagulation, was the only biomarker that showed partial mediation (proportion of mediation, 10 [1 to 28] \%).

Conclusion: Little evidence was found that the association between age and mortality in ARDS patients is mediated through age-dependent differences in host response pathways. Only tissue plasminogen activator was identified as a possible mediator of interest.

Trial registration: This trial was registered at ClinicalTrials.gov (identifier NCT01905033, date of registration July 23, 2013).

Keywords: Critical care, intensive care, ARDS, host response, Aging, Outcome, Biomarker, Mediation 


\section{Introduction}

Epidemiological data and preclinical studies using animal models of lung injury show that advanced age is associated with increased susceptibility to develop the acute respiratory distress syndrome (ARDS) [1-3]. Age has also been recognized as one of the major determinants predicting morbidity and mortality in patients with ARDS [4, 5]. Even though elderly patients usually have more comorbidities, this does only partially explain their higher burden of disease [1]. In view of the growing number of elderly patients in intensive care units (ICUs) [6], recognition as well as understanding of the association between advanced age and adverse outcome in ARDS patients could improve prognostication and may even allow for development of age-specific treatment strategies.

Responses to tissue injury in general are modified by the process of aging $[7,8]$. In healthy humans, age-related changes in the immune system, including an increased release of pro-inflammatory cytokines and chemokines [7-9], coagulation factors [10] and acute phase reactants, dysregulation of the activation and migration of inflammatory cells $[11,12]$, and endothelial dysfunction [13] have all been associated with functional decline and increased mortality. In addition, there is some evidence that this chronic activation of the "aged immune system" results in an uncontrolled host response to injury, an impairment to mount pathogens, and an inability to resolve tissue damage [8]. Little is known about the impact of this sometimes called "inflamm-aging" or "immunosenesence" on development and progression of ARDS [14]. Preclinical studies have shown a progressive pro-inflammatory status and an altered response to direct and indirect pulmonary insults in elderly animals $[3,15,16]$, yet no studies in ARDS patients have been performed to confirm this in the human setting. Those clinical studies that did compare levels of inflammation and coagulation between age groups focused on specific subgroups of critically ill patients and showed conflicting results [17-23]. In particular, none of these studies investigated age-related differences in the host response as a potential mediator of the outcome. Studying the biological host response using a statistical mediation analysis can provide an etiological understanding of the association between age and outcomes in ARDS patients [24].

This study aimed to characterize and compared the systemic host response in ARDS patients in three age groups and investigated whether the association between age and mortality in ARDS patients is mediated through age-dependent differences in host responses. It was hypothesized that an aggravated host response, i.e., a host response with an excessive increase of one or more systemic biomarkers known to be involved in inflammation, endothelial activation, or coagulation, at least partially explains the increased mortality in elderly with ARDS.

\section{Methods}

\section{Study design and setting}

This study was performed as a preplanned secondary analysis of the "Molecular Diagnosis and Risk stratification of Sepsis" (MARS) Biobank project, a prospective observational cohort study performed in the mixed ICUs of two university-affiliated hospitals in the Netherlands (ClinicalTrials.gov identifier NCT01905033) [25-29]. The Institutional Review Board of both study centers approved the study protocol (protocol no. 10-056C) with an opt-out informed consent method. Part of the patients' cohort and 
results of biomarkers' measurements have been used in previous reports on the MARS study [25-34].

\section{Inclusion and exclusion criteria}

The parent MARS study included consecutive patients admitted to the participating ICUs if expected to stay in the unit beyond the following calendar day. The current study restricted participation to patients having acute lung injury (ALI) or ARDS, according to the American-European Consensus Conference (AECC) criteria [35] as used at the time the MARS study ran. Trained researchers collected data prospectively and screened all patients daily for the presence of ALI/ARDS. After closing the MARS study, patients were re-classified as having mild, moderate, or severe ARDS, according to the Berlin definition [36], based on the $\mathrm{PaO}_{2}$ to $\mathrm{FiO}_{2}$ ratio at the day of ARDS diagnosis. Patients with a limitation on life-sustaining measures at ICU admission were excluded, patients in whom biomarkers were not determined, as were patients aged under 18 years.

\section{Age groups}

The cohort of patients was divided into three groups using cut-off levels based on agetertiles in the total cohort: young adults (18 to 54 years), middle-aged adults (55 to 67 years), and elderly ( $>67$ years and older).

\section{Endpoints}

The primary endpoint was 90-day mortality, defined as death within 90 days after the onset of ARDS. Secondary outcome measures were 30-day mortality, 1-year mortality, ICU mortality, in-hospital mortality, ventilator-free days and alive at day 28 (VFD), ICU length of stay (ICU-LOS), ICU-free days and alive at day 30 (ICU-FD), in-hospital length of stay (hosp-LOS) and hospital-free days, and alive at day 90 (hosp-FD). The occurrence of death was recorded prospectively if patients died at the ICU. In addition, for all patients, we assessed the vital status in the government registration of persons at 1 year after admission to the ICU. In case a patient was deceased, the specific date of death was recorded. Other patient data was collected prospectively, for details on data collection and definitions, see Additional file 1.

\section{Biomarker measurements}

Daily left-over EDTA anti-coagulated plasma was harvested from blood obtained for regular patient care $[28,29]$. For the current analysis, the sample nearest to the day of ARDS diagnosis was used. A panel of 20 biomarkers known to be involved in inflammation, endothelial activation, and coagulation pathways (e.g., interleukin (IL)-6, IL-8, IL-10, IL-1 $\beta$, tumor necrosis factor-alpha (TNF- $\alpha$ ), interferon-gamma (INF- $\gamma)$, intracellular adhesion molecule (ICAM)-1, matrix metalloproteinase (MMP)-8, metallopeptidase inhibitor (TIMP)-1, fractalkine, E-selectin, P-selectin, angiopoietin-1 (ANG1) and ANG2, platelet factor 4 (PF4), protein C, plasminogen activator inhibitor (PAI)-1, antithrombin (AT), D-dimer, and tissue plasminogen activator (tPA) were measured [37-39]. For assays, see Additional file 1 and Additional file 1: Table S1. 


\section{Statistical analysis}

First, clinical characteristics and outcomes were compared for the three age groups. Data are presented as absolute numbers with proportions, medians with interquartile ranges, or means with standard deviations, as appropriate. Differences between the age groups were analyzed using Kruskal-Wallis test for continuous variables and chisquared for categorical variables. Survival differences between age groups were visualized by Kaplan-Meier plots and tested with a log-rank test.

Next, the association between age and mortality was analyzed by univariate and multiple logistic regression, the confounders included are described later in the "Methods" section. Then, the host response was compared for the levels of the 20 biomarkers between young adults and middle-aged adults, and young adults and elderly using Mann-Whitney $U$ tests.

Finally, a mediation analysis was performed to investigate whether the association between the age groups and 90-day mortality was mediated through age-related differences in systemic biomarker levels (mediation step 1, Fig. 1). The mediation analysis is explained in more detail in Additional file 1. Briefly, first, the association between biomarker levels and 90-day mortality was tested using logistic regression models per each biomarker (mediation step 2). Then, the associations between the age-group and biomarker levels were determined with linear models (mediation step 3). In case of a significant association between age and a biomarker, and between that biomarker and

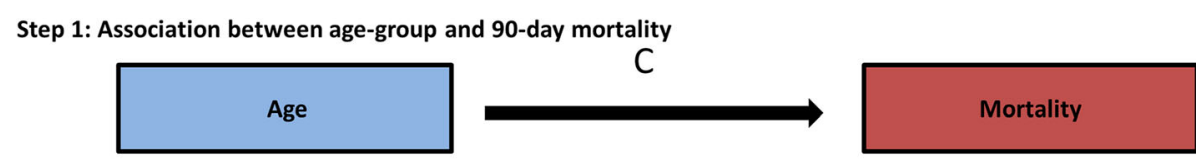

Step 2: Association between biomarkers and 90-day mortality

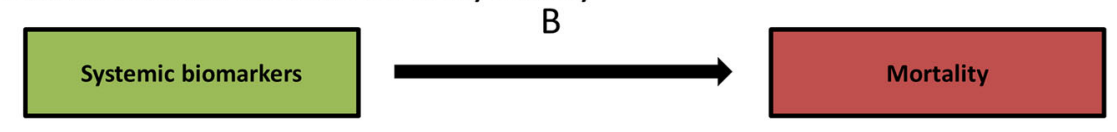

Step 3: Association between age-group and biomarkers

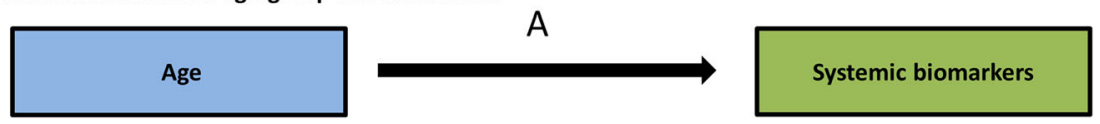

Step 4: Is the association between age-group and 90-day mortality mediated by systemic biomarkers

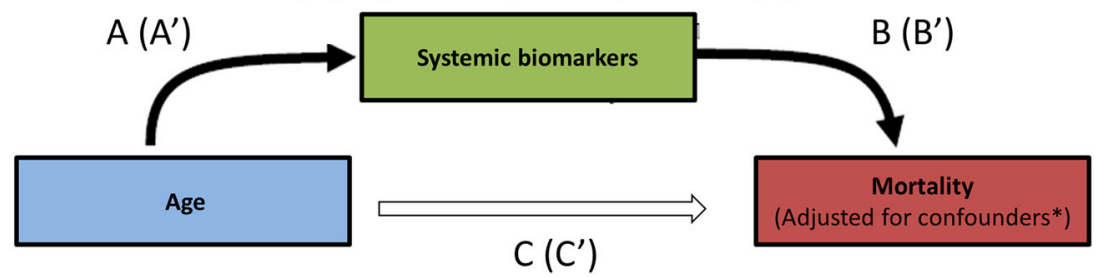

Fig. 1 Pathway analysis: stepwise mediation analysis assessing whether the association between age and mortality is mediated by age-dependent differences in biomarker levels. *Adjusted for ethnic background, gender, admission type, readmission, direct hit for ARDS, Charlson Comorbidity Index, APACHE-IV score adjusted for age, immunodeficiency, tidal volume per predicted body weight, positive end-expiratory pressure. $C=$ average direct effect $(A D E) ; A^{*} B=$ average causal mediation effect (ACME). $A^{\prime}, B^{\prime}$, and $C^{\prime}$-adjusted for confounders 
mortality, the average direct effect (ADE) of age on a 90-day mortality and the effect of age on a 90-day mortality mediated by the biomarker were modeled (average causal mediation effect, ACME) (mediation step 4). Mediation was expressed as the ratio ACME to the total effect, so-called proportion of mediation. The $95 \%$ confidence intervals of the effects were obtained via bootstrapping. Mediation was only considered relevant if the point estimate of the mediated effect was in the same direction as the total effect because "negative mediation" can per definition not explain the increased mortality [40]. A priori $<5 \%$ was considered as a small proportion of mediation, $5-20 \%$ as a moderate and $>20 \%$ as large proportions of mediation [40,41]. Young adults were defined as the reference group. Biomarkers were log-transformed to obtain normally distributed variables, and no outlines were removed from the analysis. All associations were determined with both univariate and multiple regression models, and effects were expressed as odds ratios (OR) or $\beta$-coefficients with $95 \%$ confidence intervals [95\% CI], as appropriate. For multiple linear regression models, we reported the adjusted $R^{2}$. In multiple logistic regression models, the goodness of fit was tested with the omnibus test. A $p$ value $<0.05$ was considered lack of fit.

Because an etiological mediation model was built, it was important to adjust for confounders of the exposure-outcome relation (age and mortality) and the mediator-outcome relation (biomarker levels and mortality) [40]. Potential confounders were selected according to biological plausibility, including ethnic background, gender, admission type, readmission, direct-hit for ARDS, Charlson Comorbidity Index (CCI), Acute Physiology and Chronic Health Evaluation (APACHE)-IV score adjusted for age, immunodeficiency, tidal volume per predicted body weight, and positive end-expiratory pressure. These confounders were included in all models [42]. Collinearity was tested using the variance inflation factor. A value $>2$ was considered to be collinear. If so, one of the covariates was restricted from the analyses. The handling of missing data is outlined in Additional file 1: Table S2. Of note, in a large proportion of patients the plasma concentrations of the cytokines TNF- $\alpha$ (64\%), IL-1 $\beta$ (39\%), and INF- $\gamma$ (46\%) were under the detection limit and were imputed with the LOD value which may introduce over- or underestimation of the estimates (see online Additional file 1: Table S1).

Several sensitivity analyses were performed. To potentially reduce the heterogeneity, the mediation analysis was repeated with a subgroup of patients with a direct hit for ARDS (i.e., pulmonary ARDS). In addition, because it can be argued that $\mathrm{CCI}$ and APACHE-VI are mediators instead of confounders, the adjusted mediation analysis was repeated without these variables as confounders. Furthermore, the mediation analysis was repeated with age as a continuous variable-for this analysis also, the goodness of fit was reported. Finally, the biomarker levels from a subset of patients with a sample collected at a later timepoint during ICU admission (4 to 10 days after the onset of ARDS) were explored, by comparing the median biomarker levels between the age-groups using Mann-Whitney $U$ tests. Of note, the median time to sampling from the onset of ARDS did not differ according to age (see Additional file 1: Table S22).

No statistical power analysis was conducted prior to the study. The sample size was based on the available number of patients. All statistical tests were two-tailed and were performed in $R$ statistics using the $R$-studio interface (www.r-project.org). The mediation analysis was performed using the "mediation package" [43]. In addition, a 
Benjamini-Hochberg correction for multiple comparisons was performed. A $p$ value < 0.05 was considered as statistically significant.

\section{Results}

Of 818 ARDS patients included in MARS, two patients with an age under 18 years, 106 patients with a limitation on life-sustaining measures at ICU admission, and 92 patients without a blood sample were excluded, leaving 618 subjects for the full analysis (Fig. 2). Table 1 shows patient characteristics of 209 young, 213 middleaged, and 196 elderly ARDS patients. In young patients, the prevalence of comorbidities was lower (see online Additional file 1: Table S3), but the severity of these chronic diseases among these patients was higher as reflected by a similar CCI among the age groups. In addition, young patients more frequently had an immune deficiency (see Additional file 1: Table S3).

The 90-day mortality rate was 36\% (225/618) for the entire cohort, 30\% (63/209) in young patients, 37\% (78/213) in middle-aged patients, and 43\% (84/196) in elderly patients. Middle-aged and elderly patients had 1.5 to 2.1 times higher risk of dying within 90 days after the onset of ARDS compared to young patients, after adjustment for confounders (Table 2, see online Additional file 1: Figure S1). Similar age-related differences existed for 1-year mortality, in-hospital mortality, and hospFD (Table 2,

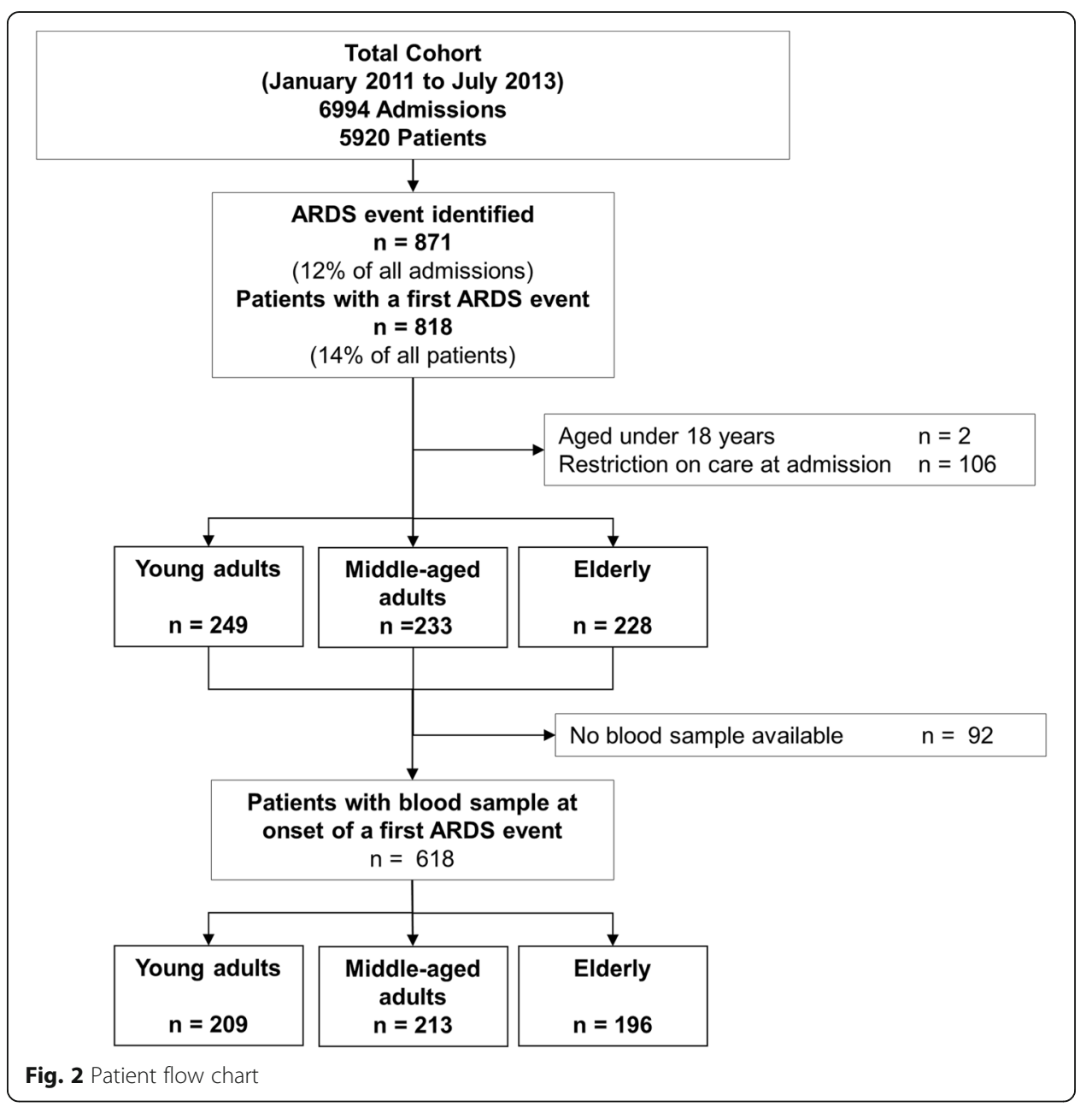




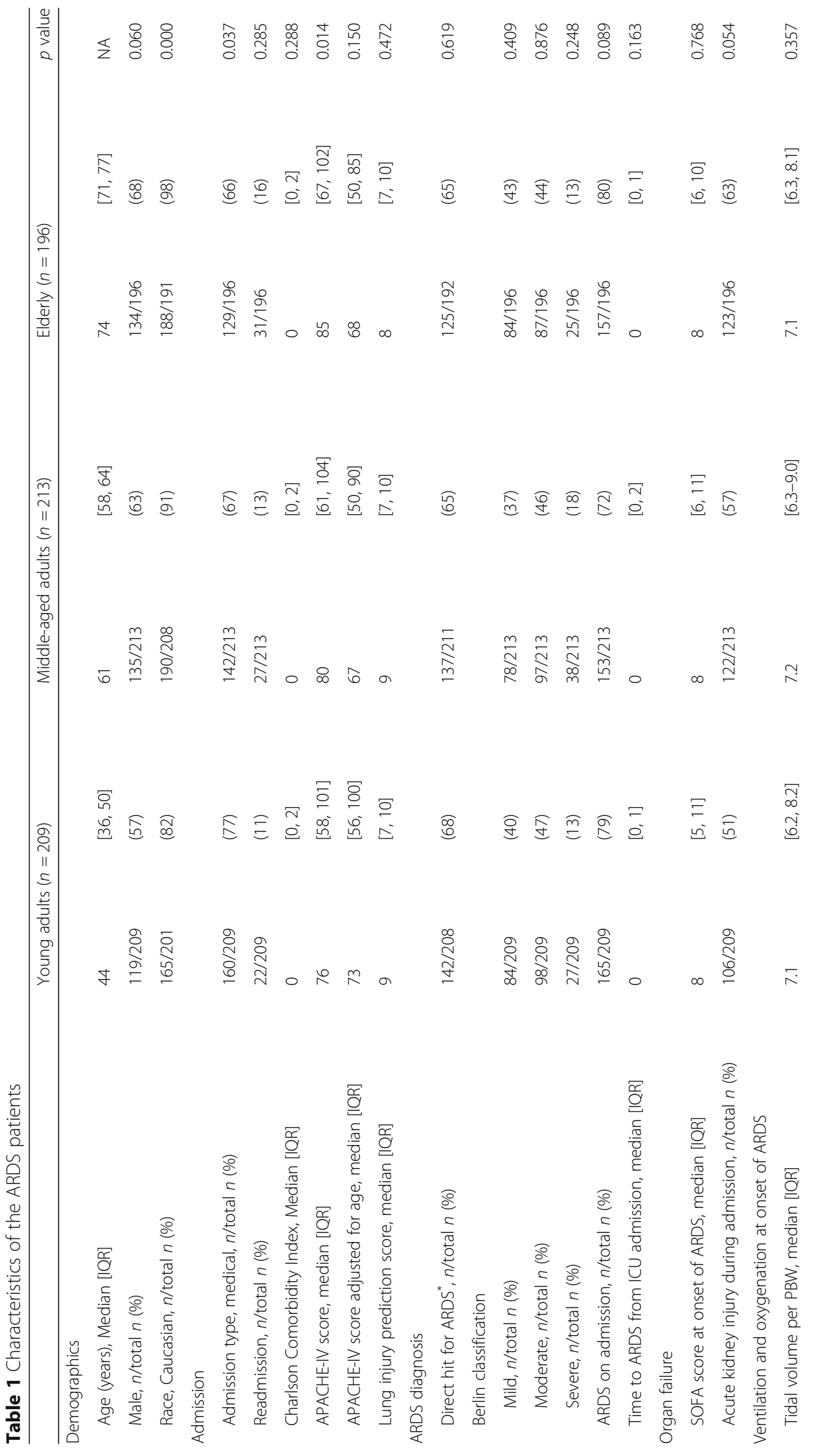




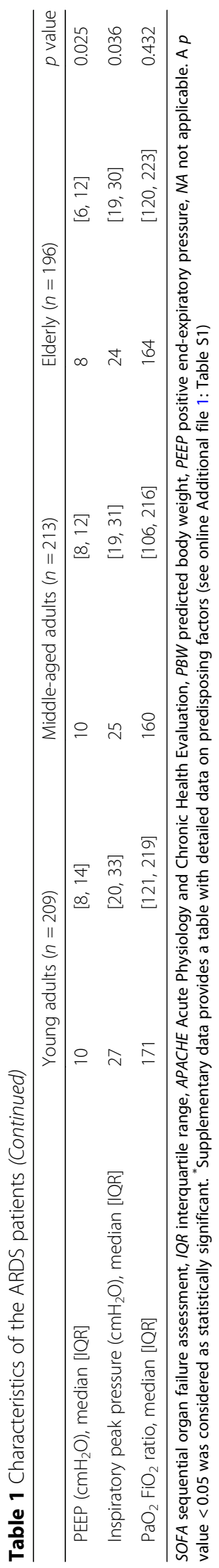




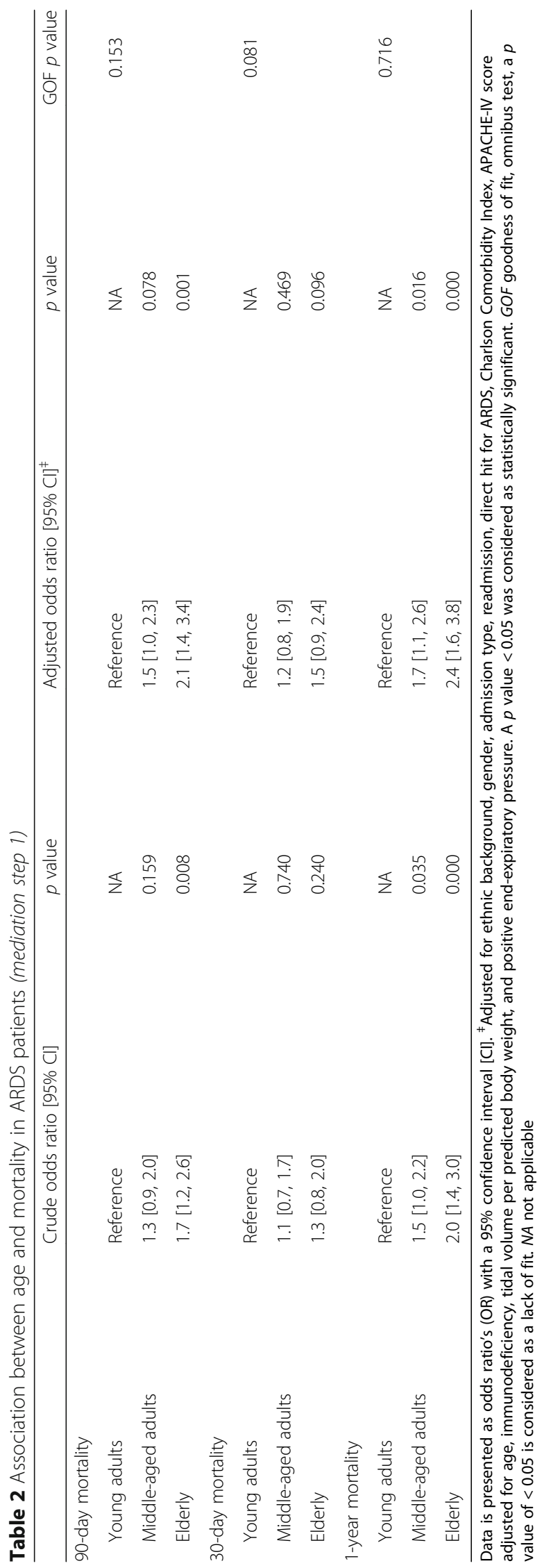


Additional file 1: Figure S1, Table S4, Table S5). There were no significant differences in ICU mortality, 30-day mortality, VFD, ICU-LOS, ICU-FD, and hosp-LOS (see Additional file 1: Table S4, Table S5). Of note, the SOFA scores and the limitation on lifesustaining measures at the time of death of the patients that died in the ICU did not differ according to age (see Additional file 1: Table S6).

Except for lower plasma levels of E-selectin and IL-10, no statistically significant differences in biomarker levels in plasma were found between young and middle-aged patients (Fig. 3). However, compared to young patients, elderly patients had lower plasma

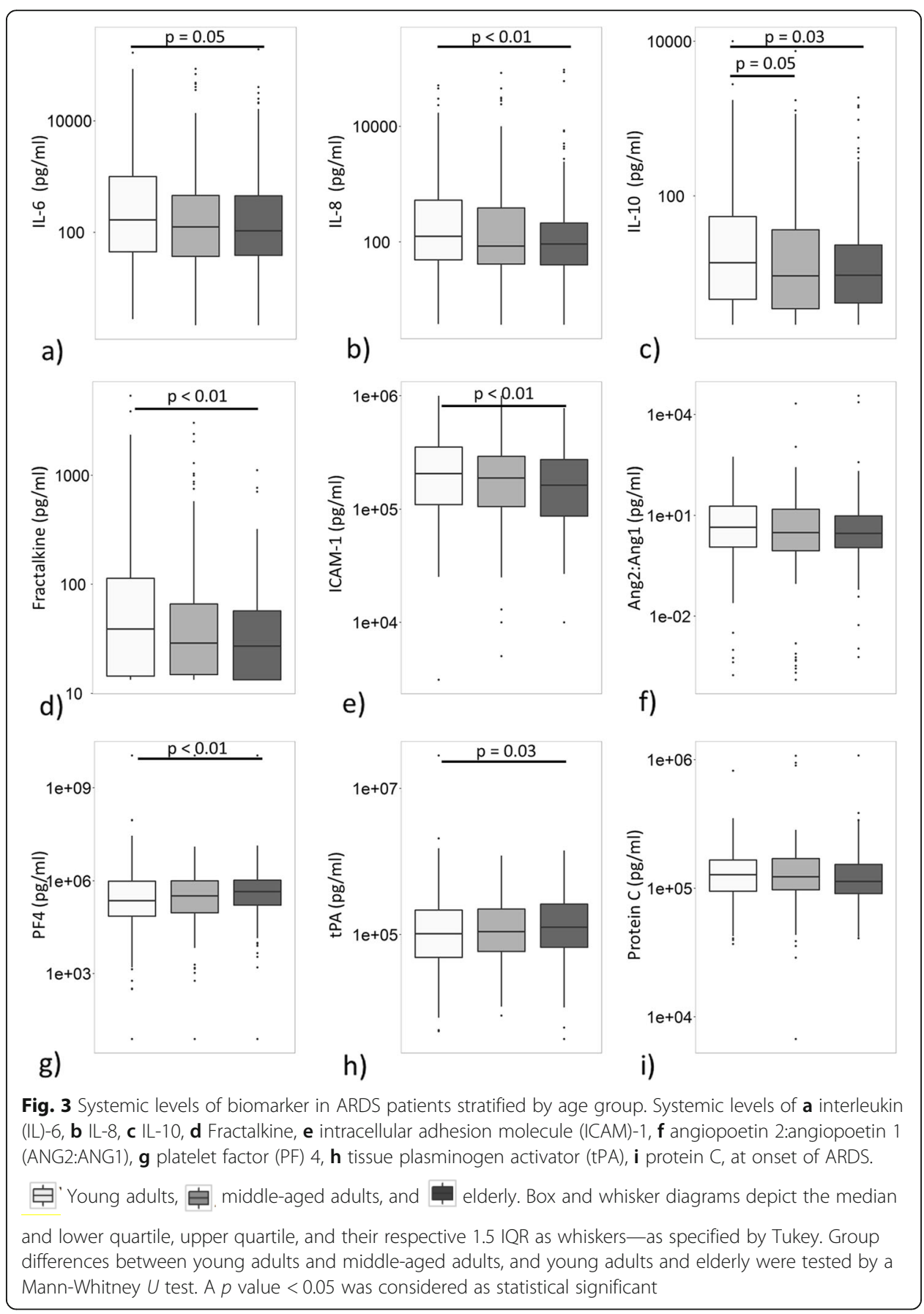


levels of IL-6, IL-8, IL-10, INF- $\gamma$, fractalkine, ICAM-1, E-selectin, and higher plasma levels of PF4 and tPA (Fig. 3, see Additional file 1: Figure S2).

Increased plasma levels of IL-8, IL-10, fractalkine, ANG2:ANG1, tPA, and PAI-1 were independently associated with 90-day mortality (see Additional file 1: Table S7). After adjusting for potential confounders, age was only statistically significant associated with increased levels of tPA and decreased levels of fractalkine and E-selectin (Tables 3, 4, and 5).

Table 3 Association between age and inflammatory marker levels in ARDS patients (mediation step 3)

\begin{tabular}{|c|c|c|c|c|c|}
\hline & Coefficient [95\% Cl] & $p$ value & Coefficient $[95 \% \mathrm{CI}]^{*}$ & $p$ value & Adjusted $R^{2}$ \\
\hline \multicolumn{6}{|l|}{ IL-6 } \\
\hline Young adults & Reference & NA & Reference & NA & 0.152 \\
\hline Middle-aged adults & $-0.33[-0.73,0.08]$ & 0.113 & $-0.30[-0.69,0.06]$ & 0.117 & \\
\hline Elderly & $-0.40[-0.81,0.01]$ & 0.057 & $-0.33[-0.73,0.06]$ & 0.100 & \\
\hline \multicolumn{6}{|l|}{ IL-8 } \\
\hline Young adults & Reference & NA & Reference & NA & 0.247 \\
\hline Middle-aged adults & $-019[-0.54,0.16]$ & 0.478 & $-0.06[-0.37,0.26]$ & 0.767 & \\
\hline Elderly & $-0.48[-0.84,-0.12]$ & 0.019 & $-0.25[-0.58,0.07]$ & 0.148 & \\
\hline \multicolumn{6}{|l|}{ IL-10 } \\
\hline Young adults & Reference & NA & Reference & NA & 0.203 \\
\hline Middle-aged adults & $-0.30[-0.62,1.02]$ & 0.174 & $-0.21[-0.51,0.08]$ & 0.133 & \\
\hline Elderly & $-0.42[-0.75,-0.09]$ & 0.032 & $-0.26[-0.57,0.04]$ & 0.058 & \\
\hline \multicolumn{6}{|l|}{$\mathrm{IL}-1 \beta$} \\
\hline Young adults & Reference & NA & Reference & NA & 0.053 \\
\hline Middle-aged adults & $-0.06[-0.20,0.09]$ & 0.461 & $-0.01[-0.16,0.14]$ & 0.854 & \\
\hline Elderly & $-0.00[-0.15,0.15]$ & 0.957 & $0.07[-0.09,0.22]$ & 0.392 & \\
\hline \multicolumn{6}{|l|}{ TNF-a } \\
\hline Young adults & Reference & NA & Reference & NA & 0.009 \\
\hline Middle-aged adults & $-0.04[-0.17,0.08]$ & 0.522 & $-0.02[-0.15,0.11]$ & 0.671 & \\
\hline Elderly & $0.05[-0.08,0.18]$ & 0.434 & $0.08[-0.06,0.22]$ & 0.248 & \\
\hline \multicolumn{6}{|l|}{ INF- $\gamma$} \\
\hline Young adults & Reference & NA & Reference & NA & 0.031 \\
\hline Middle-aged adults & $-0.16[-0.45,0.12]$ & 0.262 & $-0.11[-0.39,0.18]$ & 0.524 & \\
\hline Elderly & $-0.35[-0.65,-0.06]$ & 0.017 & $-0.24[-0.55,0.06]$ & 0.127 & \\
\hline \multicolumn{6}{|l|}{ MMP-8 } \\
\hline Young adults & Reference & NA & Reference & NA & 0.044 \\
\hline Middle-aged adults & $-0.23[-0.63,0.18]$ & 0.271 & $-0.19[-0.60,0.21]$ & 0.356 & \\
\hline Elderly & $-0.03[-0.45,0.38]$ & 0.869 & $-0.01[-0.40,0.41]$ & 0.959 & \\
\hline \multicolumn{6}{|l|}{ TIMP-1 } \\
\hline Young adults & Reference & NA & Reference & NA & 0.111 \\
\hline Middle-aged adults & $-0.08[-0.34,0.18]$ & 0.537 & $-0.03[-0.29,0.21]$ & 0.776 & \\
\hline Elderly & $-0.13[-0.40,0.13]$ & 0.328 & $0.00[-0.26,0.26]$ & 0.996 & \\
\hline
\end{tabular}

Data is presented as beta-coefficient $(\beta)$ with a $95 \%$ confidence interval [Cl]. All biomarkers are log-transformed. *Adjusted for ethnic background, gender, admission type, readmission, direct hit for ARDS, Charlson Comorbidity Index, APACHE-IV score adjusted for age, immunodeficiency, tidal volume per predicted body weight, and positive endexpiratory pressure. A $p$ value $<0.05$ was considered as statistically significant. IL interleukin, TNF- $a$ tumor necrosis factor alpha, INF- $\gamma$, interferon gamma, MMP-8 matrix metalloproteinase-8, and TIMP-1 metallopeptidase inhibitor-1. Goodness of fit, adjusted $R^{2}$. NA not applicable 
Table 4 Association between age and endothelial activation marker levels in ARDS patients (mediation step 3)

\begin{tabular}{|c|c|c|c|c|c|}
\hline & Coefficient [95\% Cl] & $p$ value & Coefficient [95\% CI] & $p$ value & Adjusted $R^{2}$ \\
\hline \multicolumn{6}{|l|}{ Fractalkine } \\
\hline Young adults & Reference & NA & Reference & NA & 0.193 \\
\hline Middle-aged adults & $-0.26[-0.49,-0.04]$ & 0.021 & $-0.17[-0.38,0.03]$ & 0.103 & \\
\hline Elderly & $-0.46[-0.69,-0.23]$ & $<0.001$ & $-0.26[-0.48,-0.05]$ & 0.018 & \\
\hline \multicolumn{6}{|l|}{ E-selectin } \\
\hline Young adults & Reference & NA & Reference & NA & 0.048 \\
\hline Middle-aged adults & $-0.31[-0.53,-0.10]$ & 0.005 & $-0.27[-0.48,-0.04]$ & 0.013 & \\
\hline Elderly & $-0.24[-0.46,-0.02]$ & 0.032 & $-0.21[-0.44,0.02]$ & 0.064 & \\
\hline \multicolumn{6}{|l|}{ P-selectin } \\
\hline Young adults & Reference & NA & Reference & NA & 0.047 \\
\hline Middle-aged adults & $-0.14[-0.36,0.08]$ & 0.214 & $-0.09[-0.30,0.13]$ & 0.510 & \\
\hline Elderly & $0.03[-0.20,0.25]$ & 0.818 & $0.05[-0.18,0.27]$ & 0.661 & \\
\hline \multicolumn{6}{|l|}{ ICAM-1 } \\
\hline Young adults & Reference & NA & Reference & NA & 0.073 \\
\hline Middle-aged adults & $-0.11[-0.26,0.04]$ & 0.168 & $-0.05[-0.20,0.10]$ & 0.547 & \\
\hline Elderly & $-0.22[-0.37,-0.07]$ & 0.005 & $-0.14[-0.29,0.02]$ & 0.073 & \\
\hline \multicolumn{6}{|l|}{ Ang2:Ang1 } \\
\hline Young adults & Reference & NA & Reference & NA & 0.046 \\
\hline Middle-aged adults & $-0.28[-0.73,0.18]$ & 0.232 & $-0.29[-0.75,0.15]$ & 0.208 & \\
\hline Elderly & $-0.22[-0.68,0.25]$ & 0.359 & $-0.19[-0.67,0.28]$ & 0.441 & \\
\hline
\end{tabular}

Data is presented as beta-coefficient $(\beta)$ with a $95 \%$ confidence interval $[\mathrm{Cl}]$. All biomarkers are log-transformed. *Adjusted for ethnic background, gender, admission type, readmission, direct hit for ARDS, Charlson Comorbidity Index, APACHE-IV score adjusted for age, immunodeficiency, tidal volume per predicted body weight, and positive endexpiratory pressure. A $p$ value $<0.05$ was considered as statistically significant. ICAM-1 intracellular adhesion molecule-1, ANG-2:ANG1 angiopoetin-2:angiopoetin-1. Goodness of fit, adjusted $R^{2}$. NA not applicable

Because age was significantly associated with IL-8, IL-10, fractalkine, PF4, PAI-1, and tPA (mediation step 2), and these biomarkers were significantly associated mortality (mediation step 3), either univariate or adjusted, this subset of biomarkers was tested as potential mediators attributing to the association between age and mortality (see Additional file 1: Table S8, Additional file 1: Table S9). Only tPA was found to be a significant mediator, mediating 10 [1 to 28$] \%(p=0.018)$ of the association between age and mortality (Fig. 4). This means that the increased risk of death in the elderly 90 days after the onset of ARDS is partially explained by their higher systemic levels of tPA. After a Benjamini-Hochberg correction for multiple testing, the proportion of mediation by tPA did not remain significant $(p=0.120)$.

The sensitivity analysis on patients with pulmonary ARDS and the adjusted analysis exclusion of CCI and APACHE-IV as covariates showed similar results, but no significant mediation in the subgroup of pulmonary ARDS (see Additional file 1: Table S10S14 and Additional file 1: Table S15-S18). The sensitivity analysis with age as a continuous variable also showed a similar result, with statistically significant partial mediation of 9 [ 1 to 26$] \%(p=0.027)$ by tPA after adjustment for confounders (see Additional file 1: Table S19-21).

Last, the subgroup analysis of 350 patients (119 young adults, 110 middle-aged adults, and 121 elderly) with a sample at a later timepoint showed that compared to young 
Table 5 Association between age and coagulation marker levels in ARDS patients (mediation step 3)

\begin{tabular}{|c|c|c|c|c|c|}
\hline & Coefficient $[95 \% \mathrm{Cl}]$ & $p$ value & Coefficient $[95 \% \mathrm{CI}]^{*}$ & $p$ value & Adjusted $R^{2}$ \\
\hline \multicolumn{6}{|l|}{ Platelet factor 4} \\
\hline Young adults & Reference & NA & Reference & NA & 0.138 \\
\hline Middle-aged adults & $0.26[-0.24,0.76]$ & 0.313 & $0.26[-0.21,0.74]$ & 0.262 & \\
\hline Elderly & $0.59[0.05,1.08]$ & 0.033 & $0.36[-0.14,0.87]$ & 0.129 & \\
\hline \multicolumn{6}{|l|}{ D-Dimer } \\
\hline Young adults & Reference & NA & Reference & NA & 0.031 \\
\hline Middle-aged adults & $-0.02[-0.22,0.19]$ & 0.857 & $-0.01[-0.22,0.20]$ & 0.942 & \\
\hline Elderly & $0.02[-0.19,0.23]$ & 0.829 & $0.03[-0.19,0.24]$ & 0.870 & \\
\hline \multicolumn{6}{|c|}{ Tissue plasminogen activator } \\
\hline Young adults & Reference & NA & Reference & NA & 0.063 \\
\hline Middle-aged adults & $0.05[-0.15,0.26]$ & 0.520 & $0.08[-0.13,0.28]$ & 0.459 & \\
\hline Elderly & $0.18[-0.04,1.39]$ & 0.103 & $0.24[0.02,0.45]$ & 0.032 & \\
\hline \multicolumn{6}{|l|}{ PAI-1 } \\
\hline Young adults & Reference & NA & Reference & NA & 0.141 \\
\hline Middle-aged adults & $-0.31[-0.64,0.03]$ & 0.072 & $-0.21[-0.54,0.10]$ & 0.141 & \\
\hline Elderly & $-0.33[-0.68,0.01]$ & 0.054 & $-0.21[-0.54,0.12]$ & 0.177 & \\
\hline \multicolumn{6}{|l|}{ Protein C } \\
\hline Young adults & Reference & NA & Reference & NA & 0.035 \\
\hline Middle-aged adults & $-0.00[-0.09,0.09]$ & 0.989 & $0.00[-0.09,0.09]$ & 0.973 & \\
\hline Elderly & $-0.05[-0.15,0.04]$ & 0.265 & $-0.07[-0.17,0.03]$ & 0.204 & \\
\hline \multicolumn{6}{|l|}{ Anti-thrombin } \\
\hline Young adults & Reference & NA & Reference & NA & 0.066 \\
\hline Middle-aged adults & $-0.00[-0.13,0.12]$ & 0.992 & $0.01[-0.12,0.13]$ & 0.850 & \\
\hline Elderly & $-0.04[-0.82,0.09]$ & 0.564 & $-0.03[-0.16,0.10]$ & 0.295 & \\
\hline
\end{tabular}

Data is presented as beta-coefficient $(\beta)$ with a 95\% confidence interval [CI]. All biomarkers are log-transformed.

${ }^{*}$ Adjusted for ethnic background, gender, admission type, readmission, direct hit for ARDS, Charlson Comorbidity Index, APACHE-IV score adjusted for age, immunodeficiency, tidal volume per predicted body weight, positive end-expiratory pressure. A $p$ value $<0.05$ was considered as statistically significant. PAI-1, plasminogen activator inhibitor-1. Goodness of fit, adjusted $R^{2}$. NA not applicable

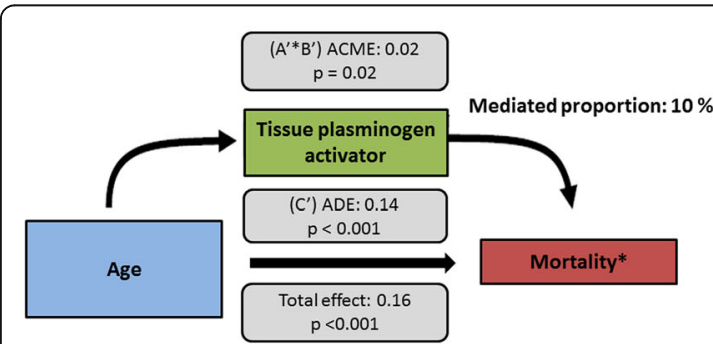

a)

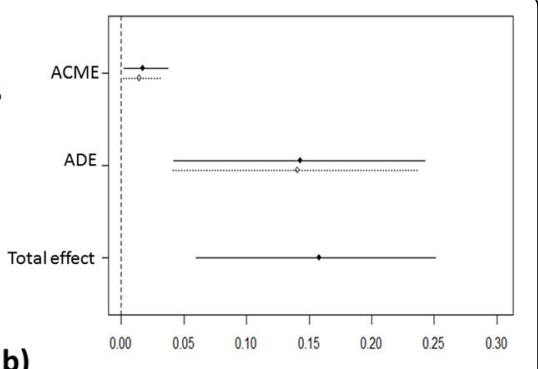

b)

Fig. 4 Mediation analysis. a, b Tissue plasminogen activator is a mediator which partially explains the association between age and mortality. *Adjusted for ethnic background, gender, admission type, readmission, direct hit for ARDS, Charlson Comorbidity Index, APACHE-IV score adjusted for age, immunodeficiency, tidal volume per predicted body weight, and positive end-expiratory pressure. ADE, average direct effect; ACME, average causal mediation effect. A $p$ value $<0.05$ was considered as statistical significant 
patients, elderly patients had again lower plasma levels of IL-6, IL-8, IL-10, INF- $\gamma$, fractalkine, ICAM-1, and MMP8 (Additional file 1: Figure S3), while plasma levels of PF4 and tPA were not found to be higher in elderly.

\section{Discussion}

In this prospective cohort of ARDS patients, a clear association was found between advanced age and higher mortality, which remained after adjustment for potential confounders including comorbidity and severity of illness scores. A mediation analysis showed only moderate $(10 \%)$ mediation of this association by tPA. In contrast to the tested hypothesis, a comparison of the inflammatory mediators and endothelial activation markers revealed lower instead of higher plasma levels in elderly compared to young patients. Advanced age was only independently associated with increased plasma levels of tPA and decreased plasma levels of fractalkine and Eselectin.

The importance of the association between age and outcome in critically ill patients has been recognized over the past decades [4, 5]. Understanding of the underlying pathophysiological mechanisms explaining this association may provide novel therapeutic targets. To our best knowledge, this is the first study in humans that investigates age-related differences in systemic host response during ARDS as a potential mediator of the increased mortality in elderly by using a mediation analysis.

Although tPA was the only marker showing mediation and the higher levels of tPA in elderly explained just $10 \%$ of the association between age and outcome, this finding deserves attention. Increased levels of tPA were strongly associated with mortality and were significantly higher in elderly. Moreover, PAI-1, the endogenous inhibitor of tPA, tended to be lower in elderly, thereby shifting the balance tPA/ PAI-1 towards fibrinolysis. Interesting in this context, recent studies have shown that both fibrinolysis shutdown and hyper-fibrinolysis are associated with increased mortality after acute injury [44, 45]. Besides its contribution to fibrinolysis, tPA has been shown to play a role in various other mechanisms involved in the pathophysiology of ARDS, such as the turnover of extracellular matrix components and extravasation of neutrophils [46, 47] [48]. Taking together, tPA could be a potential "modifiable mediator" of interest. However, it must be stressed that we performed multiple mediation models and the partial mediation of tPA did not remain significant after correction for multiple testing. Thus, this effect maybe a false discovery due to a type- 1 error. Therefore, it is important that future studies confirm a role of tPA in the association between age and outcome.

The current data showed no evidence of mediation though an enhanced inflammatory mediator response or endothelial activation. Instead, the elderly had lower plasma levels of these biomarkers compared to young patients. Lower levels of systemic inflammatory markers in critically ill elderly have previously been described and could indicate an inadequate host response (so-called immunosenescence) [17]. Nevertheless, so far no association between immunosenescence and an adverse outcome of critically ill elderly has been described [17]. Of note, the mediation analysis showed that the lower biomarker levels found in elderly were not explanatory for the observed increased mortality in this age group. However, as multiple 
aspects of immunosenescence are known to be mediated via the cellular immune response, which was not included in the current analysis, a role of immunosenescence cannot be ruled out [49]. In contrast to immunosenescence, the lower levels of inflammatory markers in elderly may also reflect differences in the precipitating injury. But, beside transplantation, we found no significant differences in predisposing factors between the age groups. Furthermore, it is important to realize that the host response is a dynamic process. We assessed the host response at the onset of ARDS, i.e., in the early phase of ARDS, while the differences in mortality seem to occur much later (e.g., 90-day mortality and 1-year mortality). Potential agedependent differences in the host response at a later time point could exist and may also explain outcome differences. Studies have suggested that a prolonged inflammatory response is associated with an adverse outcome [50, 51]. Moreover, there is evidence of age-dependent temporal differences in the host response in critically ill patients (e.g., a hyper-inflammatory response during the later phase of disease and a prolonged inflammation in elderly) [17, 20, 52-56]. The sensitivity analysis of a subset of patients with a sample at a later timepoint did not show any evidence for such prolonged inflammation in elderly. Nevertheless, serial sampling should be included in the design of future studies to assess the presence and implication of time-related differences and their relation to outcome in elderly ARDS patients. In addition, age-related differences in mechanisms involving resolution and repair of damage may also be important [57], especially, as the major differences between younger and elderly patients appear to concern the long-term outcome. Unfortunately, these mechanisms were not studied here due to the restriction in samples available for this analysis.

Despite the unambiguous evidence from various animal models of acute injury, clinical studies-including the current study-failed to detect a robust age-related difference in the systemic host response system that can explain the adverse outcome of elderly with ARDS (see Additional file 1: Table S23). Instead, the data from these clinical studies indicates that elderly ARDS patients die of other reasons than an overwhelming systemic host response. An important contributor to outcome in elderly may be "frailty," which is characterized by the vulnerability to an acute stressor [58], and its interaction with physiological reserve, i.e., the ability to maintain and restore vital functions $[59,60]$. Therefore, the relation between the biomarker levels and outcome may differ according to age. This could have important consequences for the use of biomarkers and biomarker profiles for prognostication, prediction, and selection of patients for clinical trials.

One strength of this study is its prospective design and the use of a broad range of biomarkers characterizing the three main pathways involved in the host response to injury during ARDS. However, study limitations also need to be considered. First, age-groups cut-offs were based on age-tertiles of the total ICU population. These chronological boundaries may not reflect the accurate biological stages of aging [61], although the sensitivity analysis with age as a continuous variable showed similar results. Second, the current study is a secondary analysis of a subset of patients in the parental MARS project, performed on the available dataset. Thus, the absence of statistical significance may be due to a lack of power. In addition, the included ARDS patients formed a heterogeneous patient population. 
This heterogeneity may have increased the variation in the host response, thereby limiting the ability to detect subtle influences of age-related differences on outcome. However, the subgroup analysis of patients with pulmonary ARDS did not change the results. Moreover, our cohort is relatively large compared to previous studies (see Additional file 1: Table S23), which implies more precision of the estimates. Strikingly, some of the biomarkers showed significant "negative mediation." This could be a statistical artifact (e.g., unidentified confounding or a result of multiple testing), but a biological cause of this so-called inconsistent mediation (i.e., a suppressor effect) cannot be excluded [62]. Still, because negative mediation can per definition not explain the increased mortality in elderly further exploration of this negative mediation is beyond the scope of our study. Third, despite the strict protocol for timely handling and storage of the daily left-over EDTA, the use of discarded blood by itself could have influenced the biomarker level. It should also be noted, though, that all samples were handled in the same way and irrespective of the age of patients. Fourth, we investigated only the systemic biomarkers. The pulmonary response might be different from the systemic response and more representative for mechanisms contributing to the outcome of ARDS [16]. In addition, it was not possible to determine indices of the activation of the cellular immune response as we had only daily left-over EDTA anti-coagulated plasma samples available for analysis. This is another limitation of the study because there is growing evidence that the cellular immune response plays an important role in the regulation of the inflammatory response and the progression of ARDS [63]. Moreover, instead of looking at single markers, we may have to use a broader approach like genomics and proteomics or combine biomarkers into profiles-by methods like cluster or class analysis-to identify the biological subtypes, so-called "phenotypes" $[29,64]$. It has been shown that clinical outcomes and responses to treatment depend on the phenotype of the patient [64]. Whether or not such phenotypes are depending on age or differ between different age groups need further attention.

Finally, the lack of information on the causes and circumstances of death is an important limitation of our study. We assessed the host response at an early stage of disease while death occurs much later in time. Consequentially, patients may have died of other factors than the progression of their initial disease. Previous studies have indicated that age is one of the determinants in end-of-life decisions [65]. Age-related differences in the limitation on life-sustaining measures may have introduced selection bias, which can prevent the detection of a biological relation between the host response and outcome. To minimize this influence of age-related decision-making patients with a limitation on life-sustaining measures at admission were excluded. Furthermore, the available data from patients that died in the ICU showed high respiratory and cardiovascular SOFA scores independent of age, and no age-related differences in the limitation on life-sustaining measures on the day the patients died. Still, selection bias may have influenced the outcome of the study. Last, 92 of the 818 patients with ARDS had to be excluded because there was no blood sample available at the onset of ARDS, this may have introduced selection bias. However, there was no difference in the proportion of missing samples among the age groups. 


\title{
Conclusion
}

In this cohort of ARDS patients, the observed association between advanced age and increased mortality was unrelated to alterations in systemic inflammation and endothelial cell activation. Only tPA was found to be a significant partial mediator of the association between advanced age and increased mortality, a mediation effect that disappeared after correction for multiple testing.

\section{Supplementary information}

Supplementary information accompanies this paper at https://doi.org/10.1186/s40635-019-0270-1.

Additional file 1. Online supplement data and Tables S1-S23 and Figures S1-S3

\begin{abstract}
Abbreviations
ACME: Average causal mediation effect; ADE: Average direct effect; ANG: Angiopoetin; APACHE: Acute physiology and chronic health evaluation; ARDS: Acute respiratory distress syndrome; Cl: Confidence interval; ICAM: Intracellular adhesion molecule; IL: Interleukin; INF-ү: Interferon-gamma; IQR: Interquartile range; MMP-8: Matrix metalloproteinase-8; OR: Odds ratio; PAI-1: Plasminogen activator inhibitor-1; PBW: Predicted body weight; PEEP: Positive end-expiratory pressure; PF4: Platelet factor 4; SOFA: Sequential organ failure assessment; TIMP-1: Metallopeptidase inhibitor-1; TNFa: Tumor necrosis factor alpha; tPA: Tissue plasminogen activator
\end{abstract}

\section{Acknowledgments}

de Beer FM, Bos LD, Glas GJ, Horn J, Hoogendijk AJ, van Hooijdonk RT, Huson MA, van der Poll T, Scicluna B, Schouten LR, Schultz MJ, Straat M, van Vught LA, Wieske L, Wiewel MA, and Witteveen E (Amsterdam University Medical Centers, location AMC, Amsterdam, the Netherlands); and Bonten MJ, Cremer OL, Frencken JF, van de Groep K, Klein Klouwenberg PM, Koster-Brouwer ME, Ong DS, Verboom DM (University Medical Center Utrecht, Utrecht, the Netherlands)

\section{Authors' contributions}

TVdP, MJB, MJS, and MWVA designed the work; all were involved in the acquisition; LRS, LDB, and ASN were involved in the analysis and interpretation of data; LRS, MWVA, and MJS have drafted the work; all substantively revised the draft. All authors read and approved the final manuscript.

\section{Funding}

This work was supported by grant 041-201 from Center for Translational Molecular Medicine (CTMM) (http://www. ctmm.nl) for the Molecular Diagnosis and Risk stratification of Sepsis (MARS) project; LRAS is supported by a research grant (PhD Scholarship) of the Academic Medical Center, Amsterdam, the Netherlands.

\section{Availability of data and materials}

The data that support the findings of this study are available from the MARS consortium, but restrictions apply to the availability of these data, which were used under license for the current study, and so are not publicly available. Data are however available from the authors upon reasonable request and with permission of MARS consortium.

\section{Ethics approval and consent to participate}

An opt-out informed consent method was used, as approved by the local Institutional Review Boards.

Consent for publication

Not applicable.

Competing interests

The authors declare that they have no competing interests.

\section{Author details}

${ }^{1}$ Department of Pediatric Intensive Care, Academic Medical Center, University of Amsterdam, Amsterdam, the Netherlands. ${ }^{2}$ Department of Intensive Care, Academic Medical Center, University of Amsterdam, Amsterdam, The Netherlands. ${ }^{3}$ Laboratory of Experimental Intensive Care and Anesthesiology (L.E.I.C.A), Academic Medical Center, University of Amsterdam, Amsterdam, the Netherlands. ${ }^{4}$ Department of Critical Care Medicine, Hospital Israelita Albert Einstein, São Paulo, Brazil. ${ }^{5}$ Center of Experimental and Molecular Medicine (CEMM), Academic Medical Center, University of Amsterdam, Amsterdam, the Netherlands. ${ }^{6}$ Department of Medical Microbiology, University Medical Center Utrecht, Utrecht, the Netherlands. 'DDepartment of Intensive Care, University Medical Center Utrecht, Utrecht, the Netherlands. ${ }^{8}$ Mahidol-Oxford Tropical Medicine Research Unit (MORU), Mahidol University, Bangkok, Thailand.

${ }^{9}$ Department of Pediatric Intensive Care, University Medical Center Utrecht, Utrecht, the Netherlands. 
Received: 18 July 2019 Accepted: 24 September 2019

\section{Published online: 29 October 2019}

\section{References}

1. Ely EW, Wheeler AP, Thompson BT et al (2002) Recovery rate and prognosis in older persons who develop acute lung injury and the acute respiratory distress syndrome. Ann Intern Med 136:25-36. https://doi.org/10.7326/0003-4819-136-1200201010-00007.

2. Manzano F, Yuste $\mathrm{E}$, Colmenero $\mathrm{M}$ et al (2005) Incidence of acute respiratory distress syndrome and its relation to age. $J$ Crit Care 20:274-280. https://doi.org/10.1016/j.jcrc.2005.05.008.

3. Schouten LRA, Schultz MJ, van Kaam AH et al (2015) Association between maturation and aging and pulmonary responses in animal models of lung injury: a systematic review. Anesthesiology 123:389-408. https://doi.org/10.1097/ ALN.0000000000000687.

4. Villar J, Ambrós $\mathrm{A}$, Soler JA et al (2016) $\mathrm{Age}, \mathrm{PaO}_{2} / \mathrm{FIO}_{2}$, and plateau pressure score: a proposal for a simple outcome score in patients with the acute respiratory distress syndrome. Crit Care Med 44:1361-1369. https://doi.org/10.1097/ CCM.0000000000001653.

5. Herridge MS, Chu LM, Matte A et al (2016) The RECOVER program: disability risk groups and 1-year outcome after 7 or more days of mechanical ventilation. Am J Respir Crit Care Med 194:831-844. https://doi.org/10.1164/rccm.201512-23430C

6. Wunsch H, Linde-Zwirble WT, Angus DC et al (2010) The epidemiology of mechanical ventilation use in the United States. Crit Care Med 38:1947-1953. https://doi.org/10.1097/CCM.0b013e3181ef4460.

7. Montgomery RR, Shaw AC (2015) Paradoxical changes in innate immunity in aging: recent progress and new directions. J Leukoc Biol 98:1-7. https://doi.org/10.1189/jlb.5MR0315-104R

8. Shaw AC, Goldstein DR, Montgomery RR (2013) Age-dependent dysregulation of innate immunity. Nat Rev Immunol 13: 875-887. https://doi.org/10.1038/nri3547

9. Ferrucci L, Harris TB, Guralnik JM et al (1999) Serum IL-6 level and the development of disability in older persons. J Am Geriatr Soc 47:639-646. https://doi.org/10.1111/j.1532-5415.1999.tb01583.x

10. Cohen HJ, Harris T, Pieper CF (2003) Coagulation and activation of inflammatory pathways in the development of functional decline and mortality in the elderly. Am J Med 114:180-187. https://doi.org/10.1016/S0002-9343(02)01484-5

11. Niwa Y, Kasama T, Miyachi Y, Kanoh T (1989) Neutrophil chemotaxis, phagocytosis and parameters of reactive oxygen species in human aging: cross-sectional and longitudinal studies. Life Sci 44:1655-1664. https://doi.org/10.1016/00243205(89)90482-7.

12. Butcher SK, Chahal H, Nayak L et al (2001) Senescence in innate immune responses: reduced neutrophil phagocytic capacity and CD16 expression in elderly humans. J Leukoc Biol 70:881-886. https://doi.org/10.1189/jlb.70.6.881.

13. Oakley R, Tharakan B (2014) Vascular hyperpermeability and aging. Aging Dis 5:114-125. https://doi.org/10.14336/AD 2014.0500114.

14. Franceschi $\mathrm{C}$, Bonafè $\mathrm{M}$, Valensin S et al (2000) Inflamm-aging. An evolutionary perspective on immunosenescence. Ann N Y Acad Sci 908:244-254. https://doi.org/10.1111/j.1749-6632.2000.tb06651.x

15. Saito H, Sherwood ER, Varma TK, Evers BM (2003) Effects of aging on mortality, hypothermia, and cytokine induction in mice with endotoxemia or sepsis. Mech Ageing Dev 124:1047-1058. https://doi.org/10.1016/j.mad.2003.08.002.

16. Turnbull IR, Clark AT, Stromberg PE et al (2009) Effects of aging on the immunopathologic response to sepsis. Crit Care Med 37:1018-1023. https://doi.org/10.1097/CCM.0b013e3181968f3a

17. Stanojicic M, Chen P, Xiu F, Jeschke MG (2016) Impaired immune response in elderly burn patients: new insights into the immune-senescence phenotype. Ann Surg 264:195-202. https://doi.org/10.1097/SLA.0000000000001408

18. Ginde AA, Blatchford PJ, Trzeciak S et al (2014) Age-related differences in biomarkers of acute inflammation during hospitalization for sepsis. Shock 42:99-107. https://doi.org/10.1097/SHK.0000000000000182.

19. Marik PE, Zaloga GP, NORASEPT II Study Investigators. North American Sepsis Trial II (2001) The effect of aging on circulating levels of proinflammatory cytokines during septic shock. Norasept II Study Investigators. J Am Geriatr Soc 49: 5-9. https://doi.org/10.1046/.1532-5415.2001.49003.x.

20. Kale $S$, Yende $S$, Kong $L$ et al (2010) The effects of age on inflammatory and coagulation-fibrinolysis response in patients hospitalized for pneumonia. PLoS One 5:e13852. https://doi.org/10.1371/journal.pone.0013852.

21. Kelly E, MacRedmond RE, Cullen G et al (2009) Community-acquired pneumonia in older patients: does age influence systemic cytokine levels in community-acquired pneumonia? Respirology 14:210-216. https://doi.org/10.1111/j.14401843.2008.01423.x

22. Glynn P, Coakley R, Kilgallen I, O'Neill S (1999) Neutrophil CD11b and soluble ICAM-1 and E-selectin in community acquired pneumonia. Eur Respir J 13:1380-1385. https://doi.org/10.1034/j.1399-3003.1999.13f24.x

23. Boldt J, Müller M, Heesen M et al (1997) Does age influence circulating adhesion molecules in the critically ill? Crit Care Med 25:95-100. https://doi.org/10.1097/00003246-199701000-00019

24. Bos LD, Schouten LR, Schultz MJ (2016) Promising but still uncertain steps towards better prediction of functional outcome in ICU patients. J Thorac dis 8:E838-40. Doi: https://doi.org/10.21037/jtd.2016.07.08

25. Klein Klouwenberg PMC, Frencken JF, Kuipers S et al (2017) Incidence, predictors, and outcomes of new-onset atrial fibrillation in critically ill patients with sepsis. A cohort study. Am J Respir Crit Care Med 195:205-211. https://doi.org/10. 1164/rccm.201603-06180C.

26. Klein Klouwenberg PMC, van Mourik MSM, Ong DSY et al (2014) Electronic implementation of a novel surveillance paradigm for ventilator-associated events. Feasibility and validation. Am J Respir Crit Care Med 189:947-955. https://doi. org/10.1164/rccm.201307-13760C

27. Geboers DGPJ, de Beer FM, Tuip-de Boer AM et al (2015) Plasma suPAR as a prognostic biological marker for ICU mortality in ARDS patients. Intensive Care Med 41:1281-1290. https://doi.org/10.1007/s00134-015-3924-9.

28. van Vught LA, Wiewel MA, Hoogendijk AJ et al (2017) The host response in patients with sepsis developing intensive care unit-acquired secondary infections. Am J Respir Crit Care Med 196:458-470. https://doi.org/10.1164/rccm.201606-1225OC.

29. Bos LD, Schouten LR, van Vught LA et al (2017) Identification and validation of distinct biological phenotypes in patients with acute respiratory distress syndrome by cluster analysis. Thorax 72:876-883. https://doi.org/10.1136/ thoraxjnl-2016-209719. 
30. Hoogendijk AJ, Wiewel M A, van Vught $L$ A, et al (2015) Plasma fractalkine is a sustained marker of disease severity and outcome in sepsis patients. Crit Care 19:412. doi: https://doi.org/10.1186/s13054-015-1125-0.

31. van Vught LA, Scicluna BP, Hoogendijk AJ et al (2016) Association of diabetes and diabetes treatment with the host response in critically ill sepsis patients. Crit Care 20:252. https://doi.org/10.1186/s13054-016-1429-8.

32. Boshuizen M, Leopold JH, Zakharkina T et al (2015) Levels of cytokines in broncho-alveolar lavage fluid, but not in plasma, are associated with levels of markers of lipid peroxidation in breath of ventilated ICU patients. J Breath Res 9 : 036010. https://doi.org/10.1088/1752-7155/9/3/036010.

33. Frencken JF, van Vught LA, Peelen LM et al (2017) An unbalanced inflammatory cytokine response is not associated with mortality following Sepsis: a prospective cohort study. Crit Care Med 45:e493-e499. https://doi.org/10.1097/CCM.0000000000002292.

34. Wiewel MA, de Stoppelaar SF, van Vught LA et al (2016) Chronic antiplatelet therapy is not associated with alterations in the presentation, outcome, or host response biomarkers during sepsis: a propensity-matched analysis. Intensive Care Med 42:352-360. https://doi.org/10.1007/s00134-015-4171-9.

35. Bernard GR, Artigas A, Brigham KL et al (1994) Report of the American-European Consensus Conference on acute respiratory distress syndrome: definitions, mechanisms, relevant outcomes, and clinical trial coordination. J Crit Care 9: 72-81. https://doi.org/10.1016/0883-9441(94)90033-7.

36. Ranieri VM, Rubenfeld GD, Thompson BT et al (2012) Acute respiratory distress syndrome: the Berlin definition. JAMA 307:2526-2533. https://doi.org/10.1001/jama.2012.5669.

37. O'Shea JJ, Murray PJ (2008) Cytokine signaling modules in inflammatory responses. Immunity 28:477-487. https://doi. org/10.1016/j.immuni.2008.03.002

38. Yang XP, Mattagajasingh S, Su S et al (2007) Fractalkine upregulates intercellular adhesion molecule-1 in endothelial cells through CX3CR1 and the Jak Stat5 pathway. Circ Res 101:1001-1008. https://doi.org/10.1161/CIRCRESAHA.107.160812.

39. Levi M, van der Poll T, Büller HR (2004) Bidirectional relation between inflammation and coagulation. Circulation 109: 2698-2704. https://doi.org/10.1161/01.CIR.0000131660.51520.9A.

40. Song Y, Huang YT, Song Y et al (2015) Birthweight, mediating biomarkers and the development of type 2 diabetes later in life: a prospective study of multi-ethnic women. Diabetologia 58:1220-1230. https:/doi.org/10.1007/s00125-014-3479-2.

41. Beulens JWJ, van der Schouw YT, Moons KGM et al (2013) Estimating the mediating effect of different biomarkers on the relation of alcohol consumption with the risk of type 2 diabetes. Ann Epidemiol 23:193-197. https://doi.org/10. 1016/j.annepidem.2012.12.014.

42. Valeri L, Vanderweele TJ (2013) Mediation analysis allowing for exposure-mediator interactions and causal interpretation: theoretical assumptions and implementation with SAS and SPSS macros. Psychol Methods 18:137-150. https://doi.org/ 10.1037/a0031034.

43. Tingley D, Yamamoto T, Hirose K et al (2014) Mediation: R package for causal mediation analysis. J Stat Softw 59:1-38. https://doi.org/10.18637/jss.v059.i05.

44. Moore HB, Moore EE, Gonzalez E et al (2014) Hyperfibrinolysis, physiologic fibrinolysis, and fibrinolysis shutdown: the spectrum of postinjury fibrinolysis and relevance to antifibrinolytic therapy. J Trauma Acute Care Surg 77:811-817; discussion 817. https://doi.org/10.1097/TA.0000000000000341.

45. Moore HB, Moore EE, Liras IN et al (2016) Acute fibrinolysis shutdown after injury occurs frequently and increases mortality: a multicenter evaluation of 2,540 severely injured patients. J Am Coll Surg 222:347-355. https://doi.org/10. 1016/j.jamcollsurg.2016.01.006.

46. Montrucchio G, Lupia E, De Martino A et al (1996) Plasmin promotes an endothelium-dependent adhesion of neutrophils. Circulation 93:2152-2160. https://doi.org/10.1161/01.CIR.93.12.2152.

47. Baramova EN, Bajou K, Remacle A et al (1997) Involvement of PA/plasmin system in the processing of pro-MMP-9 and in the second step of pro-MMP-2 activation. FEBS Lett 405:157-162. https://doi.org/10.1016/s0014-5793(97)00175-0.

48. Zhao Y, Sharma AK, LaPar DJ et al (2011) Depletion of tissue plasminogen activator attenuates lung ischemiareperfusion injury via inhibition of neutrophil extravasation. Am J Physiol Lung Cell Mol Physiol 300:L718-L729. https:// doi.org/10.1152/ajplung.00227.2010.

49. Fulop T, Larbi A, Dupuis $G$ et al (2017) Immunosenescence and inflamm-aging as two sides of the same coin: friends or foes? Front Immunol 8:1960. https://doi.org/10.3389/fimmu.2017.01960.

50. Narute P, Seam N, Tropea M et al (2017) Temporal changes in microrna expression in blood leukocytes from patients with the acute respiratory distress syndrome. Shock 47:688-695. https://doi.org/10.1097/SHK.0000000000000806.

51. Meduri GU, Headley S, Kohler G et al (1995) Persistent elevation of inflammatory cytokines predicts a poor outcome in ARDS: plasma IL-1 $\beta$ and IL-6 levels are consistent and efficient predictors of outcome over time. Chest 107:1062-1073. https://doi.org/10.1378/chest.107.4.1062.

52. Yokota Y, Wakai Y, Mine Y et al (1988) Degradation of host defenses against respiratory tract infection by Klebsiella pneumoniae in aged mice. Infect Immun 56:966-971.

53. Wen J, Li C-M, Gu L et al (2014) Aging reduces the expression of lung CINC and MCP-1 mRNA in a $P$. aeruginosa rat model of infection. Inflammation 37:933-941. https://doi.org/10.1007/s10753-014-9813-5.

54. Mares CA, Ojeda SS, Li Q et al (2010) Aged mice display an altered pulmonary host response to Francisella tularensis live vaccine strain (LVS) infections. Exp Gerontol 45:91-96. https://doi.org/10.1016/j.exger.2009.10.004.

55. Mares CA, Sharma J, Ojeda SS et al (2010) Attenuated response of aged mice to respiratory Francisella novicida is characterized by reduced cell death and absence of subsequent hypercytokinemia. PLoS One 5:e14088. https://doi.org/ 10.1371/journal.pone.0014088.

56. Pinheiro da Silva F, Zampieri FG, Barbeiro DF et al (2013) Septic shock in older people: a prospective cohort study. Immun Ageing 10:21. https://doi.org/10.1186/1742-4933-10-21.

57. López-Otín C, Blasco MA, Partridge L et al (2013) The hallmarks of aging. Cell 153:1194-1217. https://doi.org/10.1016/j. cell.2013.05.039.

58. McDermid RC, Stelfox HT, Bagshaw SM (2011) Frailty in the critically ill: a novel concept. Crit Care 15:301. https://doi.org/ 10.1186/cc9297.

59. Linge HM, Lee JY, Ochani K et al (2015) Age influences inflammatory responses, hemodynamics, and cardiac proteasome activation during acute lung injury. Exp Lung Res 41:216-227. https://doi.org/10.3109/01902148.2014 999174. 
60. Rosas GO, Zieman SJ, Donabedian M et al (2001) Augmented age-associated innate immune responses contribute to negative inotropic and lusitropic effects of lipopolysaccharide and interferon gamma. J Mol Cell Cardiol 33:1849-1859. https://doi.org/10.1006/jmcc.2001.1448.

61. Milbrandt EB, Eldadah B, Nayfield S et al (2010) Toward an integrated research agenda for critical illness in aging. Am J Respir Crit Care Med 182:995-1003. https://doi.org/10.1164/rccm.200904-0630CP

62. MacKinnon DP, Krull IL, Lockwood CM (2000) Equivalence of the mediation, confounding and suppression effect. Prev Sci 1:173-181. https://doi.org/10.1158/0008-5472.CAN-09-2490.

63. Lin S, Wu H, Wang C et al (2018) Regulatory T cells and acute lung injury: cytokines, uncontrolled inflammation, and therapeutic implications. Front Immunol 9:1-10. https://doi.org/10.3389/fimmu.2018.01545.

64. Calfee CS, Delucchi K, Parsons PE et al (2014) Subphenotypes in acute respiratory distress syndrome: latent class analysis of data from two randomised controlled trials. Lancet Respir Med 2:611-620. https://doi.org/10.1016/S22132600(14)70097-9.

65. Joynt GM, Gomersall CD, Tan P et al (2001) Prospective evaluation of patients refused admission to an intensive care unit: triage, futility and outcome. Intensive Care Med 27:1459-1465. https://doi.org/10.1007/s001340101041.

\section{Publisher's Note}

Springer Nature remains neutral with regard to jurisdictional claims in published maps and institutional affiliations.

Submit your manuscript to a SpringerOpen ${ }^{\circ}$ journal and benefit from:

- Convenient online submission

- Rigorous peer review

- Open access: articles freely available online

High visibility within the field

- Retaining the copyright to your article

Submit your next manuscript at $>$ springeropen.com 